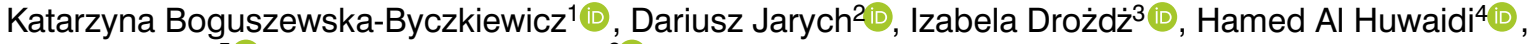
Izabela Zawlik ${ }^{5}$, Agnieszka Kołacińska ${ }^{\infty}$

${ }^{1}$ Department of Surgical Oncology, Copernicus Provincial Multidisciplinary Centre of Oncology and Traumatology, Lodz, Poland ${ }^{2}$ Laboratory of Personalized Medicine and Laboratory of Biotechnology, Bionanopark Ltd Poland, Lodz, Poland

${ }^{3}$ Department of Clinical Genetics, Medical University of Lodz, Lodz, Poland

${ }^{4}$ Medical University of Lodz, Poland

5 Department of Genetics, Institute of Medical Sciences, Laboratory of Molecular Biology, Centre for Innovative Research in Medical and Natural Sciences, Medical College, University of Rzeszow, Poland

${ }^{6}$ Department of Head and Neck Cancer Surgery, Department of Surgical Oncology, Cancer Center, Medical University of Lodz, Poland

\title{
A comparison of four commercial kits used for isolating circulating cell-free DNA: QuickGeneMINI8L (Kurabo), Maxwell RSC cfDNA Plasma Kit (Promega), cfKapture 21 Kit (MagBio), and QIAamp MinElute ccfDNA Kit (Qiagen)
}

\section{Corresponding author:}

Katarzyna Boguszewska-Byczkiewicz, Department of Surgical Oncology, Copernicus Provincial Multidisciplinary Centre of Oncology and Traumatology, 93-513 Lodz, Paderewskiego 4, Poland e-mail: k.boguszewska@kopernik.lodz.pl

Medical Research Journal 2020; Volume 5, Number 2, 92-99 10.5603/MRJ.a2020.0021 Copyright (c) 2020 Via Medica ISSN 2451-2591

\begin{abstract}
A minimally-invasive alternative to surgical biopsies is a liquid biopsy $(\mathrm{LB})$, a technique that has recently revolutionized the management of a number of tumors. One potential target biomarker of LB is cell-free DNA (cfDNA), which can act as a very sensitive indicator for certain tumors. Currently, clinical efforts are focused on increasing the quality of the cfDNA isolated for analysis.

The present study compares the efficiency of isolation by four commercial kits: QuickGeneMINI8L (Kurabo), Maxwell RSC cfDNA Plasma Kit (Promega), cfKapture 21 Kit (MagBio), and QIAamp MinElute ccfDNA Kit (Qiagen). In each case, cfDNA was isolated from three plasma samples and one serum sample. Available method for the isolation give the ability to enrich optimal diagnostic quantity of cfDNA. cfDNA can be successfully separated using all investigated kits. The greatest efficiency was demonstrated by the QIAamp MinElute ccfDNA Kit (Qiagen) and cfKapture 21 (MagBio). Large amounts of cell-free DNA can be successfully isolated from small volumes of plasma.

Key words: liquid biopsy, breast cancer, cell-free DNA
\end{abstract}

Med Res J 2020; 5 (2): 92-99

\section{Introduction}

Liquid biopsy (LB) is a minimally-invasive diagnostic method based on the isolation of biomarkers dissolved in body fluids such as blood, cerebrospinal fluid, pleura effusion, sputum, saliva or seminal fluid [1]. By analyzing various circulating molecular biomarkers, such as circulating tumor cells (CTCs), cell-free DNA (cfDNA), exosomes, proteins and microRNA(miRNA), it is possible to achieve early diagnoses of a number of diseases.
One of the most promising of these biomarkers is cell-free DNA (cfDNA), first described in 1948 [2]. The cfDNA profile corresponds with the metastatic model and disease progression; it can also reflect co-morbidity and resistance mechanisms, as well as the complete cancer gene expression profile and tumor staging [3-9]. cfDNA also includes circulating tumor DNA (ctDNA) referred to as the tumor code in the blood [10]; however, the ctDNA is mixed with a wide range of cfDNA from various sources and is hence difficult to detect clearly. In line with the recent surge of interest in the development 
of innovative technologies to detect cfDNA in LB, the present study evaluates four commercially-available cfDNA extraction kits as candidates for improved cfDNA isolation techniques.

In 2016, cfDNA testing was given FDA approval for clinical usage. Unfortunately, the current high cost of LB technology, and the associated technical challenges in cfDNA isolation, limit its clinical adoption. However, LB offers great potential for daily use as it requires little time and resources to perform, and possesses broad diagnostic potential.

Aim

The present study compares the efficiency of four cfDNA isolation kits in patients with breast cancer to determine which offers the most efficient cfDNA isolation; it also determines whether serum or plasma provides the greatest quantities of cfDNA.

\section{Material and methods}

Three patients with breast cancer were enrolled in the study; they were selected from patients hospitalized in the Department of Surgical Oncology, Copernicus Memorial Hospital, Lodz, Poland. The medical records of those patients, including the age of breast cancer removal, type of surgery, size of tumor, histological type of cancer, hormone receptor profile, grading, TNM staging and lymph node involvement, were reviewed retrospectively. Patient baseline characteristics are presented in Table 1.

All participants gave written informed consent to take part in the study. Approval for the study was given by the local ethics committee (study number RNN/ /345/15/KE dated 15.12.2015).

\section{Patient 3}

The patient was 84 years old at the time of the investigation, with lobular type breast cancer on the left side, G2, with no ductal carcinoma in situ (DCIS) component, diameter $1.8 \mathrm{~cm}$, classified as T1NO, after mastectomy. Human epidermal growth factor receptor 2 (HER2) status was negative, estrogen receptor (ER) status was positive (100\% of expression) and progesterone receptor (PR) status was positive ( $90 \%$ of expression).

\section{Patient 17}

The patient was 54 years old at the time of the investigation, with no special type (NST) breast cancer on the right side, G2 with no DCIS component, diameter $1.1 \mathrm{~cm}$, classified as $\mathrm{T} 1 \mathrm{NO}$, after mastectomy with sentinel lymph node biopsy (SLNB). HER2 status was negative, ER status was negative ( $0 \%$ of expression), PR status was negative ( $0 \%$ of expression).

\section{Patient 19}

The patient was 69 years old at the time of the investigation, with NST breast cancer on the left side, G2, with DCIS component, diameter $2 \mathrm{~cm}$, classified as T1N2 after breast conservation therapy; the patient had undergone axillary lymph node dissection.HER2 status was negative, ER status was positive, PR status was positive. The reason why we have chosen those particular 3 patients is because they fulfill the criteria of TNM class when it comes to all dimensions (size, lymph nodes and metastases).

\section{Method}

Blood samples were obtained from the peripheral veins of all participants and frozen at $-80^{\circ} \mathrm{C}$. Each serum sample was numbered according to the participating patient. The following commercial cfDNA isolating kits were analyzed: QuickGene-Mini8L (Kurabo, Japan), cfKapture 21 Kit (200-400 $\mu$ L) (MagBio Genomics, USA), Maxwell RSC ccfDNA Plasma Kit (Promega, USA), QIAamp MinElute ccfDNA (Qiagen, Germany). Laboratory testing was performed in the Laboratory of Personalized Medicine and Laboratory of Biotechnology, BioNanoPark in Lodz.

A. Isolation of cfDNA from serum sample number 3: cfDNA was isolated from serum sample 3 using two kits: QuickGeneMINI8L from Kurabo and the QIAamp MinElute ccfDNA Kit from Qiagen.

B. Isolation of cfDNA serum sample number 17: cfDNA was isolated from serum sample 17 using three kits: the aMaxwell RSC (Promega) cfDNA Plasma Kit, the cfKapture $21 \mathrm{Kit}$ (MagBio) and the QIAamp MinElute ccfDNA Kit (Qiagen).

C. Isolation of cfDNA serum sample number 19: cfDNA was isolated from serum sample 19 using four kits: QuickGeneMINI8L (Kurabo), Maxwell RSC cfDNA Plasma Kit (Promega), cfKapture 21 Kit (MagBio) and QIAamp MinElute ccfDNA Kit (Qiagen).

D. Comparison of cfDNA isolation from plasma and serum sample from patient number 19:

Isolation was carried out using two kits: the Maxwell RSC cfDNA Plasma Kit (Promega) and cfKapture 21 Kit (MagBio). Plasma and serum samples were both taken from patient number 19.

In all cases, the obtained cfDNA fragments were analyzed by automated gel electrophoresis on a Tape Station 2200( Agilent) using D1000 High Sensitive Screen Tape. 
Table 1. Patient profiles

\begin{tabular}{|c|c|c|c|c|c|c|}
\hline $\begin{array}{l}\text { Patient } \\
\text { number }\end{array}$ & $\begin{array}{c}\text { Tumor type/ } \\
\text { /DCIS } \\
\text { component }\end{array}$ & TNM/G & $\begin{array}{c}\text { Tumor size } \\
\text { [cm] }\end{array}$ & side & $\begin{array}{c}\text { HER/ } \\
\text { /ER/PR }\end{array}$ & Operation type \\
\hline 3 & Lobular/no & T1N0/G2 & 1.8 & left & $\begin{array}{c}-++/ \\
\text { /luminal A }\end{array}$ & mastectomy \\
\hline 17 & NST/no & T1N0/G2 & 1.1 & right & $\begin{array}{c}---/ \\
\text { /triple } \\
\text { negative }\end{array}$ & mastectomy \\
\hline 19 & NST/no & T1N2/G2 & 2.0 & left & -++ & $\begin{array}{c}\text { Breast } \\
\text { conservation } \\
\text { therapy }(\mathrm{BCT})\end{array}$ \\
\hline
\end{tabular}

Table 2. A comparison of the tested kits

\begin{tabular}{|c|c|c|c|}
\hline The name of a kit & Purification method & $\begin{array}{l}\text { Buffer volume } \\
{[\mu \mathrm{L}]}\end{array}$ & $\begin{array}{c}\text { Starting material volume } \\
{[\mathrm{mL}]}\end{array}$ \\
\hline QuickGeneMINI8L (Kurabo) & DNA affinity columns & 100.0 & 3.0 \\
\hline $\begin{array}{l}\text { QIAamp MinElute ccfDNA Kit } \\
\text { (Qiagen) }\end{array}$ & $\begin{array}{l}\text { magnetic beads with on- } \\
\text { column purification }\end{array}$ & 60.0 & 1.0 \\
\hline $\begin{array}{l}\text { Maxwell RSC cfDNA Plasma Kit } \\
\text { (Promega) }\end{array}$ & magnetic beads & 60.0 & 1.0 \\
\hline cfKapture 21 Kit (MagBio) & magnetic beads & 50.0 & 0.28 \\
\hline
\end{tabular}

Summary of characteristics of all kits used for isolation is shown in Table 2.

\section{Results}

A. For sample 3, a high number of cfDNA fragments about 180 bp length were observed; however, only for the QIAamp MinElute ccfDNA kit. The isolated cfDNA is presented in Figure 1.

B. For sample number 17, cfDNA was isolated successfully with all of the kits. The largest amount of cfDNA was obtained by the Qiamp MinElute ccfDNA Kit from $1 \mathrm{ml}$ of starting material. However, the highest yield, i.e. the greatest amount of cfDNA per given volume of starting material, was obtained by the cfKapture $21 \mathrm{Kit}$, as this only required $280 \mu \mathrm{L}$ of starting material. Figure 2 presents the cfDNA isolated from sample number 17 by three cfDNA isolation kits: A1 - cfKaptur 21 Kit; E1 - QIAamp MinElute ccfDNA Kit; F5 - Maxwell RSC cfDNA Plasma Kit. The efficacy of the isolation method is reflected by cfDNA concentration related to cfDNA fragment size. The horizontal axis and vertical axis represent product size and peak height of DNA concentration, respectively.

C. For sample number 19, the greatest amount of cfDNA was obtained using the Qiamp MinElute
ccfDNA Kit (i.e. from $1 \mathrm{~mL}$ material), while the highest efficiency was obtained by the cfKapture $21 \mathrm{Kit}$ (i.e. from $280 \mu \mathrm{L}$ material). The isolated cfDNA is presented in Figure 3.

D. A high level of genomic DNA contamination was observed during cfDNA isolation from serum samples, evidenced by the presence of additional products longer than $180 \mathrm{bp}$. This is confirmed by the isolation results obtained by the two kits used. In the case of cfDNA isolation from plasma, a single peak characteristic of the size of cfDNA was obtained. The isolated cfDNA is presented in Figure 4 and Figure 5.

\section{Discussion}

The concept of circulating tumor cells was first proposed by Ashworth in 1869 following the identification of tumor cells in the peripheral blood of a patient with metastatic disease. However, the relationship between cfDNA and malignant disease was only established in 1975 [16]. In recent years, a large number of studies have attempted to eliminate the background interference generated by large amounts of cfDNA from other sources: the concentration of total cfDNA in healthy individuals ranges from 0 to $100 \mathrm{ng} / \mathrm{mL}$, and this value can be as high as $1000 \mathrm{ng} / \mathrm{mL}$ in cancer patients $[17,18]$. cfDNA is 


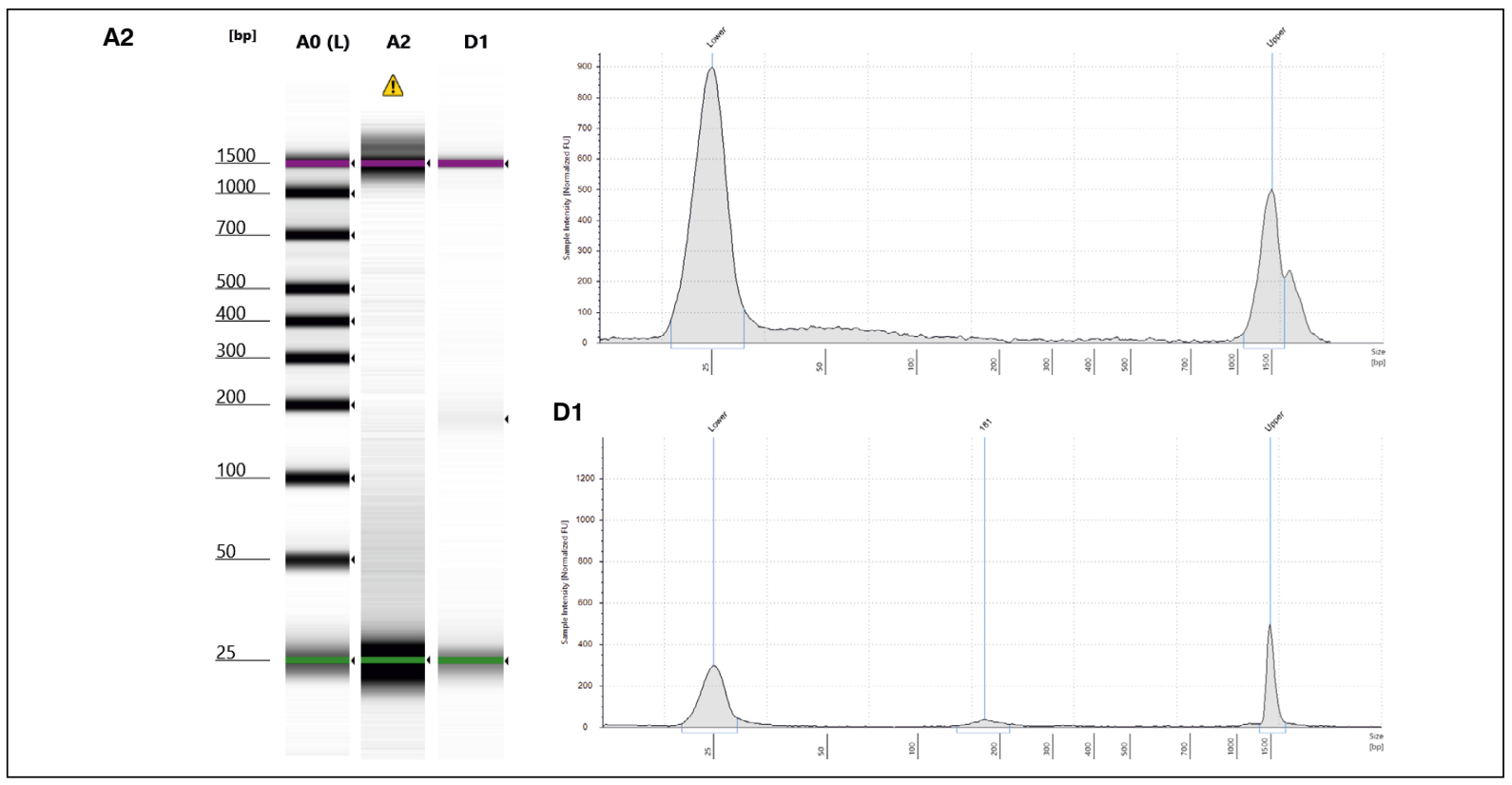

Figure 1. Electrophoresis of cfDNA isolated from sample number 3 by Agilent 2200 TapeStation 2200. A2QuickGeneMINI8L(Kurabo), D1-QIAamp MinElite ccfDNA Kit(Qiagen). The observed bands are described in more detail in Table 3. and Electropherograms of cfDNA isolation products (A2-Kurabo, D1-Qiagen), obtained with Tape Station 2200 (Agilent)

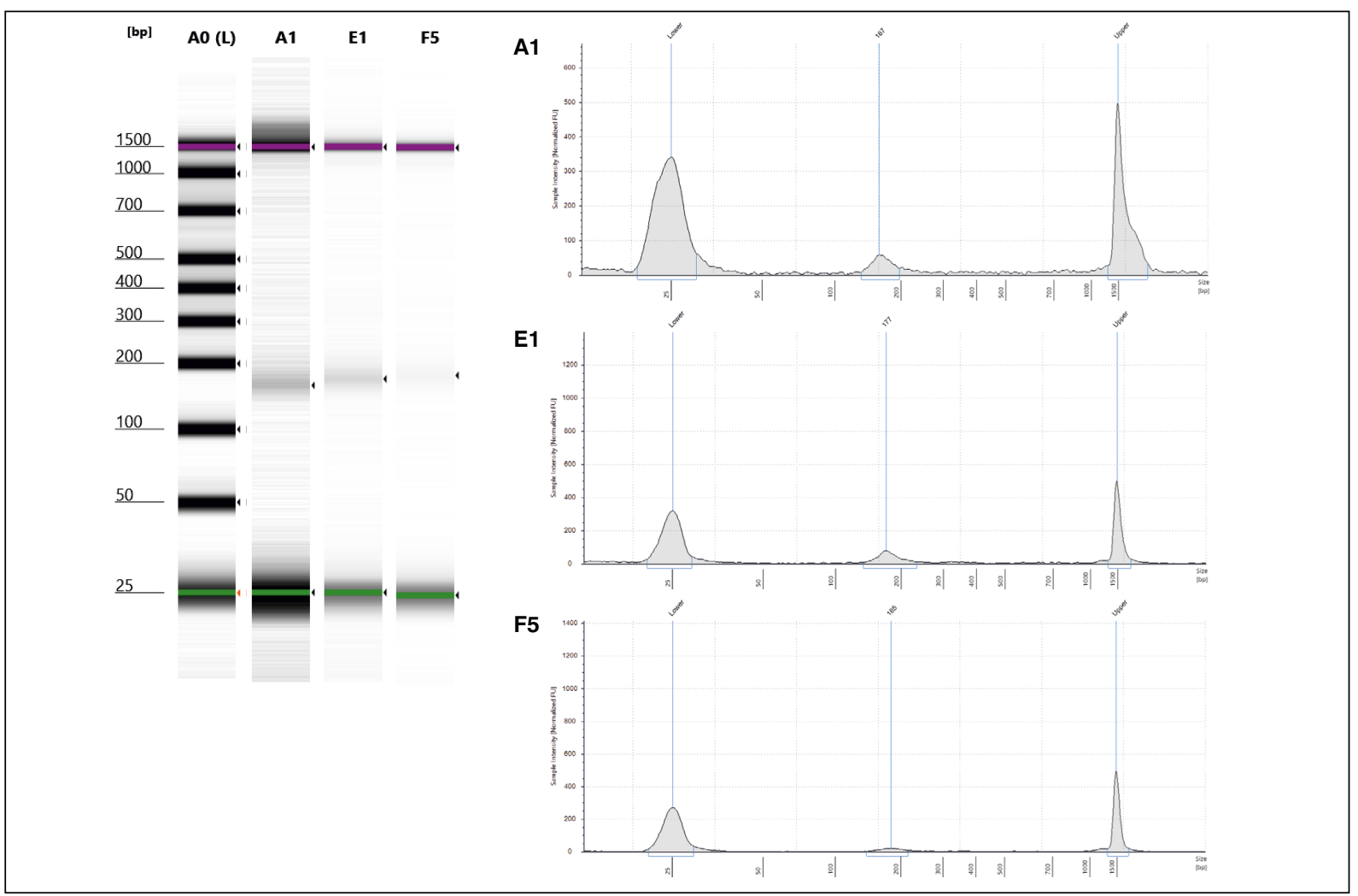

Figure 2. Electrophoresis of cfDNA isolated from sample no. 17 by Agilent 2200 TapeStation 2200. A1 - cfKapture 21 Kit; E1- QIAamp MinElute ccfDNA Kit; F5- Maxwell RSC cfDNA Plasma Kit. The observed bands are described in more detail in Table 3. and Electropherograms for cfDNA isolated fragments (A1 - cfKapture $21 \mathrm{Kit}$; E1 — QIAamp MinElute ccfDNA Kit; F5 - Maxwell RSC cfDNA Plasma Kit), obtained using an Agilent TapeStation 2200 


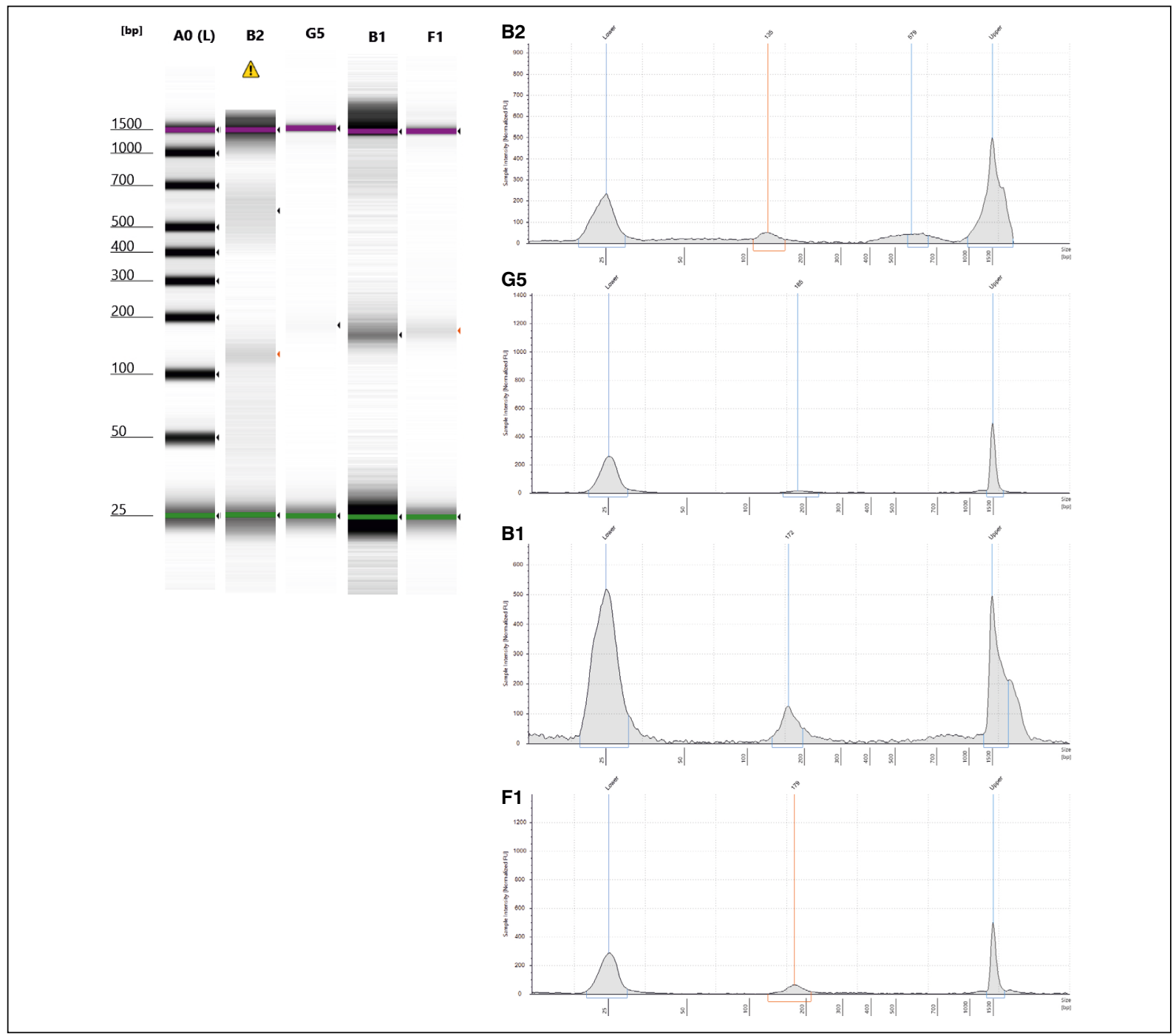

Figure 3. Electrophoresis of cfDNA isolated from sample no. 19 by Agilent 2200 TapeStation 2200: B2-QuickGeneMINI8L; G5- Maxwell RSC cfDNA Plasma Kit; B1 — cfKapture 21 Kit: F1— QIAamp MinElute ccfDNA Kit. The observed bands are described in more detail in Table 3. and Electropherograms of cfDNA isolated from sample no. 19 using the following kits: B2-QuickGeneMINI8L; G5 - Maxwell RSC cfDNA Plasma Kit; B1 - cfKapture 21 Kit: F1 - QIAamp MinElute ccfDNA Kit (TapeStation 2200, Agilent). The efficacy of the isolation method is determined by cfDNA concentration related to cfDNA fragment size. The horizontal axis and vertical axis represent the product size and peak height of DNA concentration, respectively

predominantly of hematopoietic origin, with cfDNA from non-hematopoietic cells being shorter [19, 20].

This preliminary study is intended as a segue to further analyses of isolated ctDNA. Although the approach is expensive, it has established a firm foundation for liquid biopsy (LB) as a minimally-invasive diagnostic tool with strong potential to predict clinically-important changes. Analyses performed on genetic material isolated from neoplastic cells are highly accurate and have a low false-negative rate. The presence of ctDNA can be indicative of even very low levels of potent oncogenic cells.
Our findings may play a significant role in opening this area of study. Previous studies have addressed the standardization of ctDNA measurement and the further improvement of cfDNA extraction kits, and have attempted to simplify longitudinal monitoring. The findings have yielded clinically-significant information regarding the monitoring of ctDNA quantity and accessibility.

In addition to comparing cfDNA isolation kits, the present study also compares the suitability of plasma and serum as bases for ctDNA quantification. While ctDNA is typically quantified by digital PCR (dPCR) specific for the mutations detected in the tumor tissues [9], am- 
plification refractory mutation systems (ARMSs), droplet digital PCR (ddPCR), and next-generation sequencing (NGS) can also be used [11]. In our analysis, cfDNA

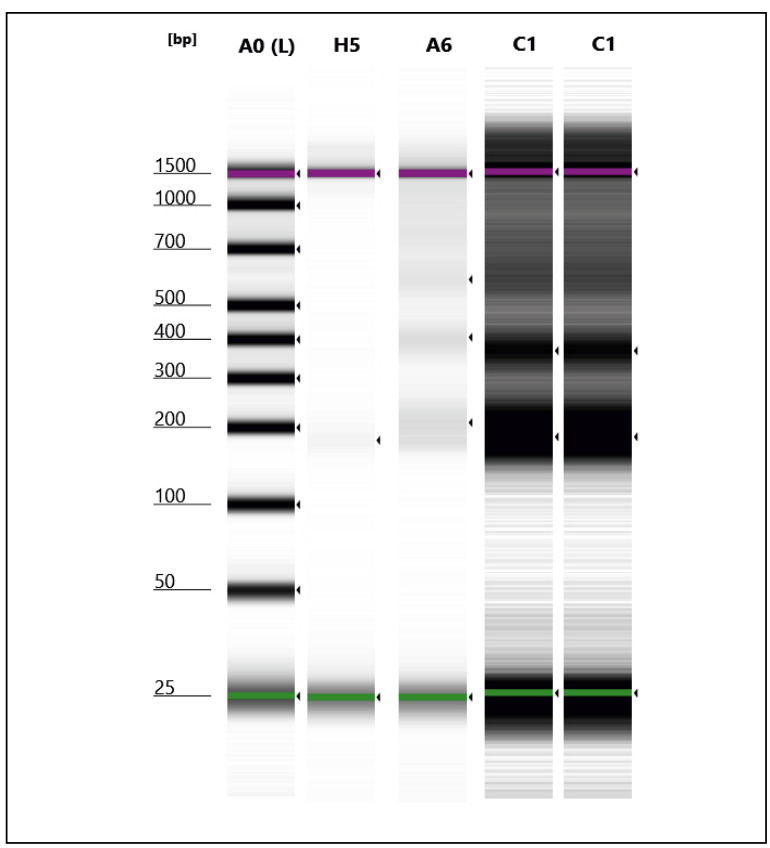

Figure 4. Electrophoresis of cfDNA isolated from sample no. 19 by Agilent 2200 TapeStation 2200. The following cfDNA were assumed to be ctDNA: $184 \mathrm{bp}$ in plasma $(\mathrm{H} 5)$ and $211 \mathrm{bp}$ in serum (A6) for the Maxwell RSC cfDNA Plasma Kit, and 185 bp in serum (C1) for the cfKapture 21 Kit. The observed bands are described in more detail in Table 4 from the peripheral blood was enriched by size-based separation: a procedure that concentrates the proportion of ctDNA within a sample based on fragment size [15]. By following this approach, a significantly higher proportion of tumor ctDNA is detected by primers that target amplicons shorter than $100 \mathrm{bp}$ and is directly correlated with the increase of ctDNA concentration; in addition, while ctDNA fragments longer than 10,000 bp are likely to originate from necrotic cells, those shorter than $1000 \mathrm{bp}$, particularly $180 \mathrm{bp}$ or multiples of this size, tend to be from apoptotic cells [12-14].

The amount of cfDNA and the length of ctDNA fragments can be used to predict progression-free survival and overall survival in patients with breast cancer. It has been demonstrated that both cfDNA fragment size and high cfDNA levels pre-treatment are associated with shorter progression-free survival and overall survival; in addition, pre-treatment cfDNA levels could independently predict prognosis for both progression-free survival and overall survival $[21,22]$. Studies of metastatic melanoma treated with immune checkpoint inhibitors found lower ctDNA concentration at baseline to be associated with shorter progression-free survival, and for the concentration to increase with tumor burden during treatment [23]. Although the amount of isolated cfDNA depends on the efficiency of the extraction method, a comparable influence is exerted by pre-analytical factors such as type of blood collection tube, centrifugation speed and storage temperature. Pre-analytical variables can also influence the release of non-mutated DNA from leucocytes, resulting in the dilution of the ctDNA fraction [24].
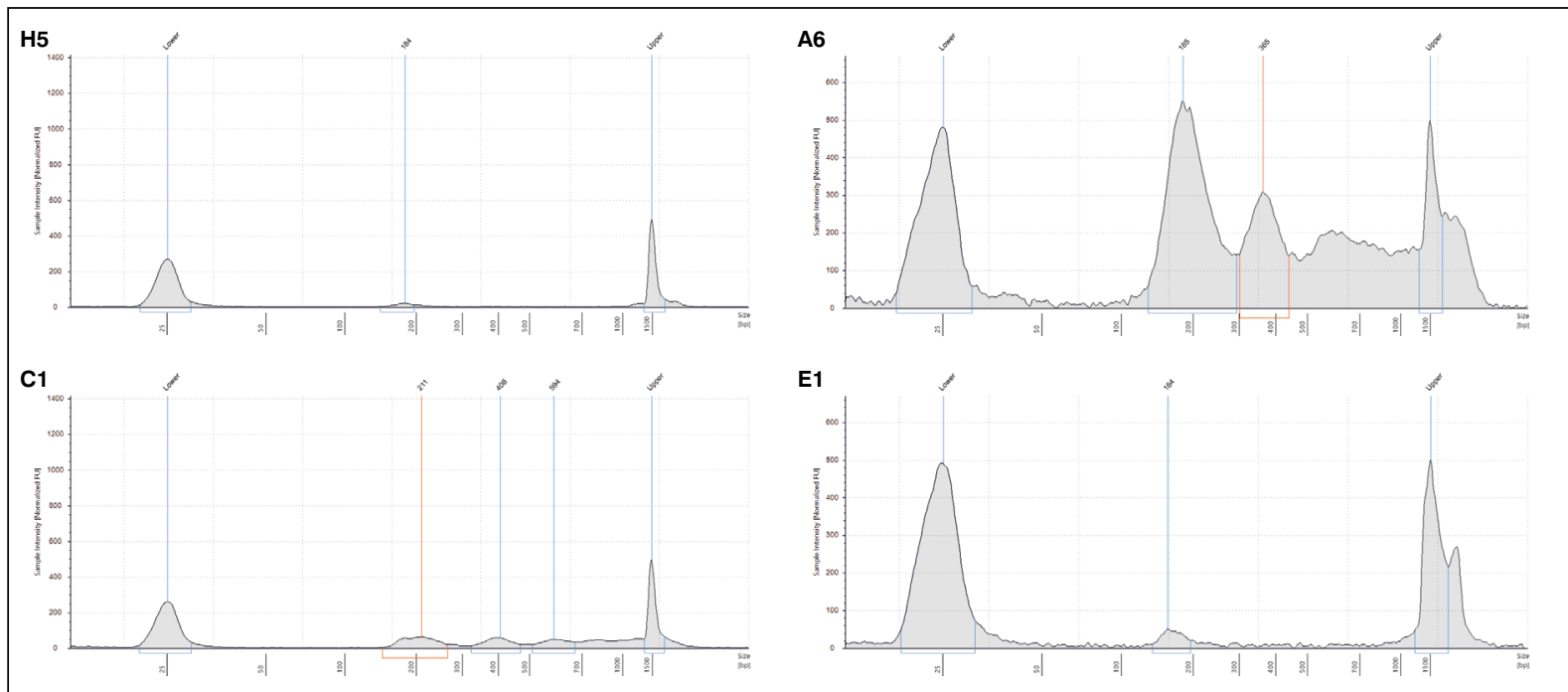

Figure 5. Electropherograms for cfDNA isolation products using the Maxwell RSC cfDNA Plasma Kit (H5 - Plasma, A6 - Serum), and cfKapture $21 \mathrm{Kit}$ (C1 - Serum, E1 - Plasma) (TapeStation 2200). The efficacy of the isolation method is reflected by cfDNA concentration related to cfDNA fragment size. The horizontal axis and vertical axis represent product size and the peak height of DNA concentration, respectively 
Table 3. Sample placement on the gel (Fig. 1, 3, 5) and a comparison of DNA concentration and fragment sizes

\begin{tabular}{lllll}
\hline $\begin{array}{l}\text { Well } \\
\text { number }\end{array}$ & $\begin{array}{l}\text { Sample } \\
\text { number }\end{array}$ & $\begin{array}{l}\text { The name of the cfDNA isolation kit } \\
\text { used }\end{array}$ & $\begin{array}{l}\text { DNA concentration } \\
{[\mathbf{p g} / \boldsymbol{\mu} \text { L] }}\end{array}$ & Fragment size [bp] \\
\hline A2 & 3 & QuickGeneMINI8L (Kurabo) & 26.8 & N/A \\
D1 & 3 & QIAamp MinElute ccfDNA Kit (Qiagen) & 59.8 & 181 \\
A1 & 17 & CfKapture 21 Kit (MagBio) & 47.7 & 167 \\
E1 & 17 & QIAamp MinElute ccfDNA Kit (Qiagen) & 107.0 & 177 \\
F5 & 17 & Maxwell RSC cfDNA Plasma Kit (Promega) & 32.8 & 185 \\
B2 & 19 & QuickGeneMINI8L (Kurabo) & 29.9 & 135 \\
G5 & 19 & Maxwell RSC cfDNA Plasma Kit (Promega) & 24.7 & 185 \\
B1 & 19 & CfKapture 21 Kit (MagBio) & 85.9 & 172 \\
F1 & 19 & QIAamp MinElute ccfDNA Kit (Qiagen) & 93.9 & 179
\end{tabular}

Table 4. Sample placement on the gel (Fig. 7) and a comparison of DNA concentration and fragment sizes

\begin{tabular}{|c|c|c|c|c|}
\hline $\begin{array}{l}\text { Well } \\
\text { number }\end{array}$ & Sample type & The name of the cfDNA isolation kit used & $\begin{array}{l}\text { DNA } \\
\text { Concentration } \\
{[\mathrm{pg} / \mu \mathrm{L}]}\end{array}$ & $\begin{array}{l}\text { Fragment } \\
\text { size [bp] }\end{array}$ \\
\hline $\mathrm{H} 5$ & Plasma & Maxwell@ RSC cfDNA Plasma Kit & 25.4 & 184 \\
\hline A6 & Serum & & $\begin{array}{l}105.0 \\
104.0 \\
89.6\end{array}$ & $\begin{array}{l}211 \\
408 \\
594\end{array}$ \\
\hline C1 & Serum & cfKapture $^{\mathrm{TM}} 21 \mathrm{Kit}$ & $\begin{array}{l}439.0 \\
378.0\end{array}$ & $\begin{array}{l}185 \\
365\end{array}$ \\
\hline E1 & Plasma & & 30.7 & 164 \\
\hline
\end{tabular}

The kits used in the present study differ with regard to the amount of starting material required. cfDNA has a short half-life of less than two hours and is cleared through the liver and kidneys; hence, failure of these organs can affect cfDNA clearance in cancer patients [25, 26]. Changes in the numbers of chromosomal regions have been detected in plasma tumor-specific cfDNA as copy number aberrations, and these can be used to compute a genomic copy number instability score [27]. The use of cfDNA offers greater clarity regarding tumor information than circulating (CTC) tumor cell analysis $[28,29]$. It is important to note that the mass of the detected tumors would typically range from $0.1 \mathrm{~g}$ to $1 \mathrm{~g}$, small enough to be present in asymptomatic individuals [30].

The quantity of cfDNA that can be extracted from plasma can vary depending on the efficiency of the chosen extraction method [30]. In the present study, cfDNA levels were established in treatment-naïve samples. Recently, cfDNA analysis has allowed a non-invasive method for the identification of resistance mutations selective for treatment method. cfDNA mutation may well serve as an early predictor of response to standard chemotherapy, and the presence of immune checkpoint inhibitors (immunotherapy) such as PD-1, PDL-1, LAG3, TIMI-3.

\section{Conclusion}

Malignant neoplasms release significant amounts of ctDNA into the circulatory system as a result of necrosis and apoptosis. Most of these fragments are in the range of $160-180 \mathrm{bp}$. These ctDNA constitutes over $90 \%$ of total circulating cfDNA. However, the amount of isolated cfDNA ranges from 10 to $100 \mathrm{ng} / \mathrm{mL}$. It is therefore important to develop an efficient isolation method. The present study compares the ability of selected kits to isolate total cfDNA, with the aim of eventually developing a method for detecting ctDNA in patients at an early stage of cancer.

The study group included a small number of patients differing in terms of age distribution, histological type of breast cancer, estrogen receptor (ER) and progesterone receptor (PR) expression status, as well as axillary lymph node metastasis and type of surgery performed: i.e. BCT vs. mastectomy. Nevertheless, the group was homogenous with respect to histological malignancy grade, this being G2, T1 in the TNM-staging system: tumor diameter 1-2 cm, no distant metastases(M0), HER2(-) negative.

Our study confirms that that cfDNA can be successfully isolated using the following kits: QuickGene-Mini8L 
(Kurabo, Japan), Catch-cfDNASerum/Plasma Kit (CatchGene, China), cfKapture 21 Kit (200-400 $\mu$ L) (MagBio Genomics, USA), Maxwell RSC ccfDNA Plasma Kit (Promega, USA) and QIAamp MinElute ccfDNA (Qiagen, Germany). Of these, the greatest efficiency was demonstrated by the QIAamp MinElute ccfDNA Kit (Qiagen) for samples 3 and 17, and cfKapture 21 for sample 19.

The efficiency of cfDNA isolation from plasma and serum samples were compared by the use of the Maxwell RSC cfDNA Plasma Kit and the cfKapture 21 Kit. A high level of genomic DNA contamination was observed in the serum samples, as evidenced by the presence of additional isolation fragments with more than $180 \mathrm{bp}$, while a single peak characteristic of cfDNA was observed in the plasma.

\section{Conflict of interest}

The authors declared that they have no conflict of interest.

Research involving Human Participants. Informed Consent was obtained.

\section{References}

1. Ponti G, Manfredini M, Tomasi A Non-blood sources of cell-free DNA for cancer molecular profiling in clinical pathology and oncology. Crit Rev Oncol Hematol. 2019; 141: 36-42, doi: 10.1016/j.critrevonc.2019.06.005, indexed in Pubmed: 31212145.

2. Mandel P, Métais P. Les acides nucléiques du plasma sanguin chez l'homme. C R Séances Soc Biol. 1948; 142: 241-243.

3. Keup C, Benyaa K, Hauch S, et al. Targeted deep sequencing revealed variants in cell-free DNA of hormone receptor-positive metastatic breast cancer patients. Cell Mol Life Sci. 2020; 77(3): 497-509, doi: 10.1007/s00018-019-03189-z, indexed in Pubmed: 31254045.

4. Shaw JA Guttery DS, Hills A et al. Mutation Analysis of Cell-Free DNA and Single Circulating Tumor Cells in Metastatic Breast Cancer Patients with High Circulating Tumor Cell Counts. Clin Cancer Res. 2017; 23(1): 88-96, doi: 10.1158/1078-0432.CCR-16-0825, indexed in Pubmed: 27334837.

5. Quandt D, Dieter Zucht H, Amann A, et al. Implementing liquid biopsies into clinical decision making for cancer immunotherapy. Oncotarget. 2017; 8(29): 48507-48520, doi: 10.18632/oncotarget.17397, indexed in Pubmed: 28501851.

6. Kim C, Paik S. Gene-expression-based prognostic assays for breast cancer. Nat Rev Clin Oncol. 2010; 7(6): 340-347, doi: 10.1038/nrclinonc.2010.61.

7. Shirkavand A, Boroujeni ZN, Aleyasin SA. Examination of methylation changes of , , , and genes in peripheral blood DNA in breast cancer patients. Indian J Cancer. 2018; 55(4): 366-371, doi: 10.4103/ijc. IJC 100 18, indexed in Pubmed: 30829272.

8. Ma $\bar{F}, G u a n, Y, Y i Z$, et al. Assessing tumor heterogeneity using ctDNA to predict and monitor therapeutic response in metastatic breast cancer. Int J Cancer. 2020; 146(5): 1359-1368, doi: 10.1002/ijc.32536, indexed in Pubmed: 31241775.

9. Cristofanilli M, Pierga JY, Reuben J, et al. The clinical use of circulating tumor cells (CTCs) enumeration for staging of metastatic breast cancer (MBC): International expert consensus paper. Crit Rev Oncol Hematol. 2019; 134: 39-45, doi: 10.1016/j.critrevonc.2018.12.004, indexed in Pubmed: 30771872.

10. Li X, Ye M, Zhang W, et al. Liquid biopsy of circulating tumor DNA and biosensor applications. Biosens Bioelectron. 2019; 126: 596-607, doi: 10.1016/j.bios.2018.11.037, indexed in Pubmed: 30502682.

11. Leon SA, Green A, Yaros MJ, et al. Radioimmunoassay for nanogram quantities of DNA. J Immunol Methods. 1975; 9(2): 157-164, doi: 10.1016/0022-1759(75)90106-4, indexed in Pubmed: 1206227
12. Gedvilaitè V, Schveigert D, Cicénas S. Cell-free DNA in non-small cell lung cancer. Acta Med Litu. 2017; 24(2): 138-144, doi: 10.6001/actamedica.v24i2.3495, indexed in Pubmed: 28845132.

13. Esposito A, Criscitiello C, Trapani D, et al. The Emerging Role of "Liquid Biopsies," Circulating Tumor Cells, and Circulating Cell-Free Tumor DNA in Lung Cancer Diagnosis and Identification of Resistance Mutations. Curr Oncol Rep. 2017; 19(1): 1, doi: 10.1007/s11912-0170564-y, indexed in Pubmed: 28110461.

14. Lui YYN, Chik KW, Chiu RWK, et al. Predominant hematopoietic origin of cell-free DNA in plasma and serum after sex-mismatched bone marrow transplantation. Clin Chem. 2002; 48(3): 421-427, indexed in Pubmed: 11861434

15. Zheng YWL, Chan KC, Sun $\mathrm{H}$, et al. Nonhematopoietically derived DNA is shorter than hematopoietically derived DNA in plasma: a transplantation model. Clin Chem. 2012; 58(3): 549-558, doi: 10.1373/clinchem.2011.169318, indexed in Pubmed: 22052939.

16. Herbreteau G, Vallée A, Knol AC, et al. Quantitative monitoring of circulating tumor DNA predicts response of cutaneous metastatic melanoma to anti-PD1 immunotherapy. Oncotarget. 2018; 9(38): 25265-25276, doi: 10.18632/oncotarget.25404, indexed in Pubmed: 29861869 .

17. Underhill HR, Kitzman JO, Hellwig S, et al. Fragment Length of Circulating Tumor DNA. PLoS Genet. 2016; 12(7): e1006162, doi: 10.1371/journal.pgen.1006162, indexed in Pubmed: 27428049.

18. Li Li, Zhang J, Jiang $X$, et al. Promising clinical application of ctDNA in evaluating immunotherapy efficacy. Am J Cancer Res. 2018; 8(10): 1947-1956, indexed in Pubmed: 30416847.

19. Mouliere F, Robert B, Arnau Peyrotte E, et al. High fragmentation characterizes tumour-derived circulating DNA. PLoS One. 2011; 6(9): e23418, doi: 10.1371/journal.pone.0023418, indexed in Pubmed 21909401

20. Jahr S, Hentze H, Englisch S, et al. DNA fragments in the blood plasma of cancer patients: quantitations and evidence for their origin from apoptotic and necrotic cells. Cancer Res. 2001; 61(4): 1659-1665, indexed in Pubmed: 11245480

21. Lapin M, Oltedal S, Tjensvoll K, et al. Fragment size and level of cell-free DNA provide prognostic information in patients with advanced pancreatic cancer. J Transl Med. 2018; 16(1): 300, doi: 10.1186/s12967018-1677-2, indexed in Pubmed: 30400802

22. Rack B, Schindlbeck C, Jückstock J, et al. SUCCESS Study Group. Circulating tumor cells predict survival in early average-to-high risk breast cancer patients. J Natl Cancer Inst. 2014; 106(5), doi: 10.1093/inci/dju066, indexed in Pubmed: 24832787.

23. Gray ES, Rizos H, Reid AL, et al. Circulating tumor DNA to monitor treatment response and detect acquired resistance in patients with metastatic melanoma. Oncotarget. 2015; 6(39): 42008-42018, doi: 10.18632/oncotarget.5788, indexed in Pubmed: 26524482.

24. Cavallone L, Aldamry M, Lafleur J, et al. A Study of Pre-Analytical Variables and Optimization of Extraction Method for Circulating Tumor DNA Measurements by Digital Droplet PCR. Cancer Epidemiol Biomarkers Prev. 2019; 28(5): 909-916, doi: 10.1158/1055-9965.EPI-18-0586, indexed in Pubmed: 30824523

25. Elshimali YI, Khaddour H, Sarkissyan M, et al. The clinical utilization of circulating cell free DNA (CCFDNA) in blood of cancer patients. Int J Mol Sci. 2013; 14(9): 18925-18958, doi: 10.3390/ijms140918925, indexed in Pubmed: 24065096.

26. Kirsch C, Weickmann S, Schmidt B, et al. An improved method for the isolation of free-circulating plasma DNA and cell-free DNA from other body fluids. Ann N Y Acad Sci. 2008; 1137: 135-139, doi: 10.1196/annals.1448.035, indexed in Pubmed: 18837937

27. Oellerich M, Schütz E, Beck J, et al. Using circulating cell-free DNA to monitor personalized cancer therapy. Crit Rev Clin Lab Sci. 2017; 54(3): 205-218, doi: 10.1080/10408363.2017.1299683, indexed in Pubmed: 28393575.

28. Freidin MB, Freydina DV, Leung $M$, et al. Circulating tumor DNA outperforms circulating tumor cells for KRAS mutation detection in thoracic malignancies. Clin Chem. 2015; 61(10): 1299-1304, doi: 10.1373/clinchem.2015.242453, indexed in Pubmed: 26272233.

29. Dawson SJ, Tsui DWY, Murtaza M, et al. Analysis of circulating tumor DNA to monitor metastatic breast cancer. N Engl J Med. 2013; 368(13) 1199-1209, doi: 10.1056/NEJMoa1213261, indexed in Pubmed 23484797

30. Fiala C, Diamandis EP. Utility of circulating tumor DNA in cancer diagnostics with emphasis on early detection. BMC Med. 2018; 16(1) 166, doi: 10.1186/s12916-018-1157-9, indexed in Pubmed: 30285732

31. Fleischhacker M, Schmidt B, Weickmann S, et al. Methods for isolation of cell-free plasma DNA strongly affect DNA yield. Clin Chim Acta. 2011; 412(23-24): 2085-2088, doi: 10.1016/j.cca.2011.07.011, indexed in Pubmed: 21861994 